\title{
PLANEJAMENTO INSTITUCIONAL EM SAÚDE: O CASO DA SUSTENTABILIDADE AMBIENTAL EM UM HOSPITAL
}

\author{
Leandro Januário de Lima ${ }^{1}$ \\ José Ferreira Lima Júnior ${ }^{2}$ \\ Roberto Ramon Queiroz de Assis ${ }^{3}$ \\ Giliara Carol Diniz de Luna Gurgel ${ }^{4}$
}

\begin{abstract}
RESUMO. O objetivo deste estudo é conhecer o planejamento institucional elaborado por um hospital universitário do sertão da Paraíba para a promoção da sustentabilidade ambiental. Dotados de uma rotina de atividades peculiar, com setores que chegam a funcionar durante 24 horas por dia, os hospitais vêm se tornando peças-chave no desenvolvimento de atitudes sustentáveis que se justificam tanto pela quantidade e qualidade dos resíduos gerados quanto pela demanda energética destas instituições. Assim, o planejamento institucional em seus três níveis de atuação - estratégico, tático e operacional - ganha destaque, pois, configura-se como mecanismo de enfrentamento da problemática ambiental que é incorporada nos determinantes sociais do processo saúdedoença. Nesse sentido, desenvolveu-se uma pesquisa exploratória, bibliográfica e documental por meio da qual foram analisados os documentos institucionais de um hospital universitário do alto sertão paraibano no que tange a questão da sustentabilidade ambiental. Concluiu-se no estudo deste caso que já estão implantadas algumas atividades e estratégias sustentáveis em alguns dos setores da organização, além de determinados ambientes apresentarem potencial de no futuro também disporem de mecanismos mais compatíveis com o desenvolvimento harmônico do planeta.
\end{abstract}

Palavras chave: Sustentabilidade; administração hospitalar; gestão em saúde.

\section{INSTITUTIONAL PLANNING IN HEALTH: THE CASE OF ENVIRONMENTAL SUSTAINABILITY IN A UNIVERSITY HOSPITAL}

\begin{abstract}
The objective of this study is to know the institutional planning prepared by a university hospital in the backlands of Paraíba to promote environmental sustainability. Endowed with a peculiar routine of activities, with sectors that come to operate for 24 hours a day, hospitals have become key players in the development of sustainable attitudes that are justified both by the quantity and quality of waste generated and by the energy demand of these institutions. Thus, institutional planning in its three levels of action - strategic, tactical and operational - is highlighted, as it is configured as a

\footnotetext{
1 Bolsista PIBIC-CNPq, graduando em Medicina, Unidade Acadêmica de Ciências da Vida/UFCG

${ }^{2}$ Curso Técnico em Saúde Bucal. Escola Técnica de Saúde de Cajazeiras. Centro de Formação de

Professores. Universidade Federal de Campina Grande.

${ }^{3}$ Graduando em História/CFP/UFCG

${ }^{4}$ Curso Técnico em Saúde Bucal. Escola Técnica de Saúde de Cajazeiras. Centro de Formação de Professores. Universidade Federal de Campina Grande.
} 
mechanism to address the environmental problem that is incorporated into the social determinants of the health-disease process. In this sense, an exploratory, bibliographical and documentary research was developed, through which the institutional documents of a university hospital of the high rural dryland of the state of Paraiba were analyzed regarding the issue of environmental sustainability. It was concluded in the study of this case that some sustainable activities and strategies are already implemented in some of the sectors of the organization, in addition to certain environments presenting potential in the future also to have mechanisms more compatible with the harmonic development of the planet.

Keywords: Sustainability; hospital administration; Management.

\section{PLANIFICACIÓN INSTITUCIONAL EN SALUD: EL CASO DE LA SUSTENTABILIDAD AMBIENTAL EN UN HOSPITAL UNIVERSITARIO}

Resumen. El objetivo de este estudio es conocer la planificación institucional elaborada por un hospital universitario del sertão de Paraíba para la promoción de la sustentabilidad ambiental. Dotados de una rutina de actividades peculiar, con sectores que llegan a funcionar durante 24 horas al día, los hospitales se están convirtiendo en piezas clave en el desarrollo de actitudes sostenibles que se justifican tanto por la cantidad y calidad de los residuos generados como por la demanda energética de estas instituciones. Así, la planificación institucional en sus tres niveles de actuación estratégico, táctico y operacional - obtiene destaque, pues, se configura como mecanismo de enfrentamiento de la problemática ambiental que se incorpora a los determinantes sociales del proceso salud-enfermedad. En ese sentido, se desarrolló una investigación exploratoria, bibliográfica y documental por medio de la cual se analizaron los documentos institucionales de un hospital universitario del alto semiarido paraibano en lo que se refiere a la cuestión de la sostenibilidad ambiental. Se concluyó en el estudio de este caso que ya están implantadas algunas actividades y estrategias sostenibles en algunos de los sectores de la organización, además de determinados ambientes presentar potencial para que en el futuro también disponer de mecanismos más compatibles con el desarrollo armónico del planeta.

Palabras clave: Sostenibilidad; Administración hospitalaria; Gestión en la salud.

\section{Introdução}

O desenvolvimento sustentável (DS) entra no cenário das políticas públicas no Brasil a partir da década de 1990, com a emergente preocupação do uso do planeta advinda da necessidade de ofertar bens e serviços a uma população crescente e, por conseguinte, um alerta do crescimento econômico; questões essas bastante difundidas em eventos internacionais como a RIO+20 que debatem os desafios do DS e a implantação de uma economia verde (BUSS et al., 2012). 
Como fruto dessa discussão erguem-se definições para o DS, cujo conceito mais aceito e mais empregado é o da Comissão Brundt-land (WCED, 1987), elaborado pela Comissão Mundial sobre o Meio Ambiente e Desenvolvimento que define como sendo aquele capaz de suprir as necessidades da população presente, sem comprometer as necessidades das gerações futuras. Várias significações são dadas ao DS, sendo que três pontos elementares as unem, sendo elas, as questões ambientais, sociais e econômicas, consideradas as dimensões do DS (CLARO; CLARO; AMÂNCIO, 2008).

Essas três dimensões na literatura científica são intituladas de triple bottom line (TBL), frequentemente empregado para o termo de sustentabilidade organizacional; são amplamente aplicados em instituições com finalidades de controlar gastos e adequar seus modos de produção, objetivando economizar e minimizar impactos ambientais; estudos como o de Campos e Ramos (2014) mostram que a interação entre esses fatores promovem resultados satisfatórios em suas dimensões.

Em um sentido mais amplo, notamos que o DS tem uma característica própria, que é pensar em longo prazo, não apenas focar nas demandas contemporâneas, levando a refletir que atitudes tomadas pela sociedade hoje terão reflexo da sociedade que vivenciaremos no futuro. Sendo assim, sustentabilidade compreende ações que garantem qualidade de vida, conservação do meio ambiente, adequação territorial; nesse foco a sustentabilidade ambiental pode ser alcançada a partir da utilização racional dos recursos renováveis de forma a garantir sua reposição, assegurar atividades produtivas menos dependentes de recursos naturais tendo em vista sua finitude, e pela distribuição equânime dos bens produzidos onde não haja acumulação prejudicial (NASCIMENTO, 2012).

Ou como sustenta Sachs (1993, p. 23):

A sustentabilidade ambiental pode ser alcançada por meio da intensificação do uso dos recursos potenciais... Para propósitos socialmente válidos; da limitação do consumo de combustíveis fósseis e de outros recursos e produtos facilmente esgotáveis ou ambientalmente prejudiciais, substituindo-se por recursos ou produtos renováveis e/ou abundantes e ambientalmente inofensivos; redução do volume de resíduos e de poluição...; intensificação da pesquisa de tecnologias limpas. 
Dada sua importância, o desenvolvimento sustentável entra em pauta nas discussões do planejamento de instituições do setor público e privado, mas como sustentam Barbieri et al. (2010), apesar dessa discussão ser mais difundida no campo institucional de produção de bens de consumo, essa emergente preocupação suscita que os diversos seguimentos se posicionem diante das premências da sustentabilidade e repensem seus modos de produção e atuação.

Nessa perspectiva, instituições de diversos setores estão incorporando a sustentabilidade em seus processos produtivos. Um exemplo recente tem sido a adoção dos princípios da sustentabilidade pela área da saúde. Nesta, um destaque particular é dada aos hospitais, que também podem ser considerados como uma empresa, os quais ofertam serviços de média e alta complexidade em saúde que englobam, segundo Dias (2004, p. 8), "tratamento, ensino, pesquisa, reabilitação, promoção da saúde e prevenção da doença".

Consoante Vilaça e Oliveira (2008, p.7), estes estabelecimentos também podem ser caracterizados quanto a sua dinâmica de funcionalidade:

Um hospital tem uma dinâmica muito específica: funcionamento ininterrupto; intensa circulação de públicos estratégicos - usuários e familiares -; exigência de instalações capazes de tornarem autônomos diversos subsistemas e criar condições reais concernentes à manutenção de tal estrutura física.

Desse modo, a grande demanda dos serviços de saúde e seus altos custos na manutenção e operacionalização das tecnologias tornam-se pertinentes às instituições de saúde agregar valores da sustentabilidade, com objetivo de minimizar impactos ambientais e redução de gastos, garantindo uma melhor oferta dos serviços de saúde, bem como sua autossuficiência, sem comprometer a qualidade do cuidado prestado à população. Em especial, as edificações hospitalares por serem consumidores potenciais de energia e recursos ambientais, bem como geradores de resíduos de fração infectante (VILAS-BOAS, 2011).

O presente trabalho busca a aproximação entre o campo da saúde e a sustentabilidade na sua dimensão ambiental, por mais que sejam considerados campos distintos, o DS complementa o campo da saúde, a partir do momento em que levamos em considerações as premências da demanda socioambiental do DS, e os processos produtivos dos hospitais, bem como sua função e relevância social. Para tanto, 
objetivou-se conhecer o planejamento institucional elaborado por um hospital universitário do sertão da Paraíba para a promoção da sustentabilidade ambiental.

\section{Método}

Foi adotada neste estudo a modalidade de pesquisa exploratória, bibliográfica e documental, que constitui uma estratégia alternativa de pesquisa empregada nos estudos desenvolvidos no campo da saúde, bem como nas ciências ambientais. Nesse método, o conhecimento trazido pelos conjuntos entre a bibliografia científica pesquisada acerca da temática, acrescido aos documentos institucionais sobre a sustentabilidade ambiental torna-se ponto de partida para reflexão e reconstrução desse conhecimento.

Documentos são compreendidos como todas as realizações produzidas pelo homem que se mostram como indícios de sua ação e que podem revelar suas ideias, opiniões e formas de atuar e viver. Nesta concepção é possível apontar vários tipos de documentos: os escritos; os numéricos ou estatísticos; os de reprodução de som e imagem; e os documentos-objeto (GIL, 2008).

A pesquisa documental trilha os mesmos caminhos da pesquisa bibliográfica, não sendo fácil por vezes distingui-las. A pesquisa bibliográfica utiliza fontes constituídas por material já elaborado, consistindo basicamente por livros e artigos científicos localizados em bibliotecas. Nesta pesquisa documental ora proposta, recorreu-se a fontes documentais de uma instituição hospitalar de assistência pública federal.

Nessa perspectiva, a coleta de documentos apresenta-se como importante fase da pesquisa documental, exigindo do pesquisador alguns cuidados e procedimentos técnicos acerca da aproximação do local onde se pretende realizar a "garimpagem" das fontes que lhes pareçam relevantes à sua investigação. Formalizar esta aproximação com intuito de esclarecer os objetivos de pesquisa e a importância desta constituiu-se um dos artifícios necessários nos primeiros contatos e, principalmente, para que o acesso aos acervos e fontes fosse autorizado. No cerne da discussão aqui apresentada, adotou-se uma abordagem qualitativa no método, enfatizando não a quantificação ou descrição dos dados recolhidos, mas a importância das informações que podem ser geradas a partir de um olhar cuidadoso e crítico das fontes documentais. Compreende-se ainda que, dependendo da área de pesquisa do investigador e dos interesses do estudo, 
documentos que podem ser desprezíveis para uns podem ocupar lugar central para outros (SILVA et al., 2009).

A pesquisa em tela está sendo realizada em um hospital situado no interior do estado da Paraíba, de caráter universitário, que abrange atendimento da população do alto sertão da Paraíba, atendendo inclusive demanda oriunda dos estados do Ceará e do Rio Grande do Norte, oferecendo serviços de emergência e urgência na especialidade de pediatria.

Fazem parte da investigação os documentos da referida instituição de saúde, quais sejam Missão, Visão e Valores da organização; Procedimentos Operacionais Padrão (POPs) de alguns setores como serviço de nutrição, gestão da assistência farmacêutica, dentre outros; Memorial Descritivo da Infraestrutura hospitalar e o Plano Diretor Estratégico do HU. Os documentos selecionados respeitam o seguinte critério de inclusão: ser documento oficial da instituição. A pesquisa contempla duas etapas, sendo a primeira, para o levantamento dos documentos institucionais que comporão o conjunto de documentos analisados; e a segunda etapa, para a análise do conteúdo.

A análise de conteúdo é para Gil (2008) a técnica mais elaborada e de maior prestígio no campo da observação documental e constitui-se como meio para estudar as comunicações entre os homens enfatizando o conteúdo das mensagens por eles emitidas. Na concepção de Bardin (2006), ela se define como um conjunto de técnicas de análise das comunicações, visando, por procedimentos sistemáticos e objetivos de descrição do conteúdo das mensagens, obter indicadores quantitativos ou não que permitam a inferência de conhecimentos relativos às condições de produção/recepção (variáveis inferidas) das mensagens.

\section{Resultados e discussões}

A pesquisa desenvolveu-se em duas frentes; a primeira, uma visita inicial de caráter exploratório para fins de conhecimento das estruturas da unidade hospitalar, compreendendo entre elas, os setores internos e estruturas externas do Hospital Universitário Júlio Bandeira (HUJB).

$\mathrm{Na}$ segunda etapa, realizou-se levantamento de documentos institucionais respeitando o critério de inclusão na pesquisa. Dentre os investigados, foram solicitados 
os referidos documentos: Memorial Descritivo da Infraestrutura Hospitalar; Plano Diretor Estratégico (PDE-2017/2018); Planta do edifício; Procedimentos Operacionais Padrões (POP’s) dos setores de farmácia e nutrição. Até o momento já foram analisados o memorial descritivo e o PDE. Logo, os resultados aqui apresentados são ainda parciais.

Tais fontes documentais foram disponibilizadas para fins de pesquisa científica e receberam tratamento analítico, sendo essa a etapa de maior relevância, compreendendo que a pesquisa propõe à investigação de documentos institucionais através da heurística, buscando dentro de tais fontes, analisar a existência de políticas que seja conducentes à sustentabilidade ambiental, sejam elas, captação e reutilização de água, adequação de estrutura física, implantação de política que fomente a conscientização ambiental dos colaboradores do HUJB e reutilização ou reciclagem de material oriundo das atividades hospitalares.

\section{Plano Diretor Estratégico (PDE)}

O PDE do HUJB-UFCG é fruto de uma parceria entre EBSERH/MEC e Hospital Sírio Libanês, por intermédio do Instituto Sírio-Libanês de Ensino e Pesquisa (IEP/HSL) que teve como objetivo principal dois nortes: capacitar de gestores de Hospitais Universitários Federais (HUF) e, posteriormente, a elaborar do PDE construído pelo mesmo, conseguintemente, fomentar competências na equipe de governança do HUF (BATISTA et al., 2016).

O PDE do HUJB foi construído na $3^{\circ}$ fase "Projeto de Capacitação e Planos Diretores Estratégicos dos Hospitais Universitários Federais - HUFs", após adesão do mesmo a EBSERH/MEC. Para sua elaboração foi levado em consideração a contextualização do hospital no ano de 2016, por meio de "Análise Situacional” de macro problemas enfrentados pelo HUF, e proposição de melhorias e soluções, que irão nortear o biênio gerencial 2017/2018 (EBSERH, 2016).

Por ser um documento idealizado a partir de ideias coletivas, compreende-se que o mesmo resguarda interesses do conjunto de atores sociais, que podem expressar o quanto de consciência sustentável existe na equipe de governança e equipe ampliada do 
hospital, podendo ser tratadas de forma direta ou indireta, sem ser mencionado o termo sustentabilidade.

Quanto à sustentabilidade ambiental e autossuficiência do HUF, a unidade hospitalar conta com uma Usina de Produção de Gases Medicinais e subestação de energia com geradores de energia elétrica, portanto, independe de fontes externas, que por sua vez, pode simbolizar uma economia financeira nestes aspectos; tornar o hospital autossuficiente nestes setores é garantir um processo produtivo menos dependente de recursos naturais, revelando aí, um aspecto da sustentabilidade ambiental e responsabilidade social, tal ponto resguardado nos valores da instituição.

Dentre os valores adotados no planejamento do biênio 2017-2018, a responsabilidade socioambiental foi nominada como um valor institucional, podendo ser caracterizado, como sendo aquelas responsabilidades que a unidade hospitalar tem com o meio ambiente e sociedade, além dos seus deveres legais nos respectivos níveis citados, visando sempre benefícios. Então, o conjunto de ações voltadas para este aspecto, compreende a capacidade de resposta aos problemas sociais e ambientais que vivenciamos (LIMA, 2007).

Em fase de mudança, na seção "Ferramentas utilizadas na construção do PDE do HUJB-UFCG” onde foram detalhadas metodologias para o diagnóstico situacional do HUJB, foi levado em consideração sete modelos de referência, um dos quais contemplou a "Administração Econômico-Financeira" com uma adesão global de 48\% "cujo objetivo foi estabelecer um conjunto de boas práticas de gestão nos hospitais, frente ao desenvolvimento de ações para a evolução do patamar administrativo e assistencial” (BATISTA et al., 2016, p. 50). Tem-se que a partir de uma gestão financeira responsável é possível atingir a responsabilidade socioambiental, pois, evita o ataque desnecessário e predatório para recursos finitos, além de garantir recursos para o bom funcionamento da unidade.

Neste contexto, o compromisso com práticas equilibradas no ambiente de trabalho surge como uma estratégia que engloba valores da gestão participativa, que envolve gestores, docentes e discentes, segundo Ceccim (2005), é um ato político que surge da necessidade de corresponder às demandas da população no setor da saúde, por meio da aprendizagem situacional vivenciada nos ambientes de trabalho. Desta problemática, vemos que se for do interesse coletivo torna-se pertinente trabalhar às 
premências da sustentabilidade ambiental dentro do HU, a educação em saúde torna-se um instrumento para a conscientização do pessoal, e na formulação de políticas institucionais que fomentem tais interesses. Esta oportunidade está nas entrelinhas do plano diretor estratégico e pode concretizar o valor institucional da sustentabilidade.

Ainda no PDE, a seção Infraestrutura Hospitalar, elucida a preocupação do crescimento versus desenvolvimento; preocupar-se com uma edificação bem elaborada pode favorecer uma melhor assistência, pois os profissionais disporão de uma estrutura adequada para suas atividades profissionais, que engloba a incorporação de novas tecnologias, bem como, garantir uma otimização do atendimento à população, com procedimentos mais eficientes, com menos custos e rapidez no diagnóstico, assim reduzindo tempo de espera. Logo, pensar em sustentabilidade, não diz respeito apenas reduzir, reciclar ou reutilizar e sim compreender toda cadeia produtiva, desde as relações internas de trabalho, planejamento, disposição dos serviços, aspectos sociais e ambientais, bem como ressaltar a importância da esfera política como um indutor de medidas que possa incentivar e apoiar iniciativas que favoreçam a sustentabilidade nas relações homem, sociedade e natureza.

\section{Memorial Descritivo da Infraestrutura Hospitalar}

O memorial descritivo da infraestrutura hospitalar é um documento que contém informações sobre distribuição e organização de setores internos e externos do HU, nominado como "MEMORIAL DESCRITIVO PROPOSTA ASSISTENCIAL REFORMA E AMPLIAÇÃO DO HUJB” as descrições contidas no documento estão em consonância com a nova proposta de reestruturação do hospital, totalizando cinco setores, que se subdividem formando ambientes, que por vez, cada um pode conter mais de uma sala, alguns ambientes ainda não estão em pleno funcionamento e algumas salas ainda não foram sequer construídas. Ao todo estão previstos 24 ambientes distribuídos entre os setores de administração, apoio ao ensino, apoio diagnóstico, unidades de cuidado e apoio terapêutico, além de apoio técnico e manutenção.

Com uma estrutura complexa dotadas de especificidades, alguns setores irão funcionar 24 horas por dia. Devido a sua dinâmica de funcionamento, eles não poderão ser interrompidos para não ocasionar prejuízos à assistência prestada à sociedade; são 
exemplos: recepção, triagem, consultório-admissão, uma sala cirúrgica, posto policial, serviço social, sala verde-observação, sala de raios-X, as enfermarias para adolescentes, lactantes e crianças, isolamento pediátrico, armazenagem e controle, unidade de laboratórios de análises clínicas e setor de coleta.

No tangente a sustentabilidade ambiental, o Memorial Descritivo traz algumas informações já contidas no PDE e, por vez, exemplifica e denota a importância da Usina de Produção de Gazes Medicinais. "A escolha pela Usina se deu por tratar-se de uma tecnologia sofisticada e com alta eficiência, apresentando-se como uma solução alternativa altamente viável e segura para o fornecimento de oxigênio e demais gases para o hospital" (HUJB, 2016). O HU conta com um gerador de energia elétrica com potência de força automática de $160 \mathrm{Kva}$. Como mencionado anteriormente, o HU é autossuficiente nestes aspectos e por vez pode compensar gastos referentes aos setores de funcionamento ininterrupto.

O memorial descritivo também expressa preocupação com a produção de resíduos oriundos das atividades hospitalares, contando com galpão de armazenamento dos contêineres, coberto, revestido internamente (piso e paredes) com material impermeável, fechado com três divisórias separado por classificação de risco A, D, e E. Grupo A, os que apresentam risco potencial à saúde pública e ao meio ambiente devido à possível presença de agentes biológicos, dentre eles, materiais contendo secreções e líquidos orgânicos; no grupo $\mathrm{D}$, os resíduos comuns e no grupo $\mathrm{E}$ os materiais perfurocortantes ou escarificantes, tais como agulhas e escalpes (BRASIL, 2005).

Ainda nesta frente, os setores com potencial de contaminação classificados como áreas críticas dispõem de estrutura e recursos que favorecem a minimização de impactos e riscos ao homem e natureza. O material epóxi está presente em todo Centro Cirúrgico/Obstétrico nas paredes, teto e piso. Esta preocupação com a biossegurança encontra a sustentabilidade ambiental no que tange a disseminação de agentes que possam causar danos a espécies animais e da flora, evitando desta forma perdas no patrimônio biológico.

A sala de Raio-X foi classificada como uma sala controlada cuja estrutura que promove a segurança da meio ambiente, profissional e paciente com paredes, piso, teto e portas com blindagem que proporcione proteção radiológica às áreas adjacentes, de acordo com os requisitos de otimização, observando o previsto na Portaria 453/1998 do 
Ministério da Saúde, que estabelece as diretrizes básicas de proteção radiológica em radiodiagnóstico médico e odontológico.

Saindo dos documentos de suporte físico e observando a realidade do hospital e das estruturas físicas do HUJB, observou-se que as ações de sustentabilidade presentes ainda são poucas e executadas de forma isolada apenas para atender necessidades, mas já sinalizam o início de uma construção coletiva de hábitos sustentáveis. As ações desenvolvidas ainda de forma tímida se justificam em face de mudanças constantes na instituição como reformas, construções de setores, adequação de estrutura física, capacitação da equipe de trabalhadores para fins de gestão futura, dentre outros.

Por fim, o hospital possui uma estrutura favorável à aplicação de ações sustentáveis, ficando definido como uma edificação com potencial de desenvolvimento sustentável, com setores e atividades onde podem ser implantadas ações sustentáveis, a exemplo dos serviços de lavanderia que ainda não reutiliza água. Por contar com uma área construída de $3.119,16 \mathrm{~m}^{2}$, há a possibilidade do favorecimento da captação de águas pluviais, além da possível implantação de painéis para captação de energia solar no futuro, por o hospital estar localizado em uma região geográfica com alta incidência solar. Em contrapartida, em cada sala ou setor do hospital já existe considerável aproveitamento da luz durante o dia com janelas de vidros transparentes que permitem a entrada de luz solar, reduzindo o consumo de luz elétrica.

\section{Considerações finais}

Embora o modelo de desenvolvimento adotado pela maioria das instituições atualmente seja danoso ao planeta, a busca pela implantação de atitudes mais sustentáveis estão se apresentando mais concretas no meio privado, sendo as atividades do setor público ainda se apresentam tímidas frente à problemática ambiental.

A área da saúde no Brasil tem uma participação muito significativa do setor público, sobretudo após a criação do Sistema Único de Saúde. As atividades deste setor também são permeadas pelas noções de sustentabilidade social, econômica e ambiental. Todas estas faces do desenvolvimento sustentável estão relacionadas ao processo saúdedoença e aos hospitais enquanto organizações exigindo uma contrapartida com propostas que visem atingi-lo, sobretudo, com um enfrentamento de ações intersetoriais e interdisciplinares.

RPI Revista de Pesquisa Interdisciplinar, Cajazeiras, v. 3, n. 1, 2-14, jan/jul. de 2018. 
$\mathrm{Na}$ instituição alvo desta pesquisa, os documentos institucionais já absorveram a necessidade de incluir a temática ambiental nos planejamentos e práticas rotineiras. $\mathrm{O}$ plano diretor estratégico e memorial descritivo apontam ações ainda insipientes, mas significativas pela iniciativa, além de setores que ainda possuem potencial e podem desempenhar ações mais sustentáveis.

Assim, avançar na implementação de políticas institucionais de cunho sustentável irá proporcionar um remodelamento dos arranjos institucionais levando à compreensão mais ampla do conceito de saúde que, por ser um fenômeno social complexo, é influenciado também pelas questões ambientais.

\section{Referências}

BARBIERI, J. C. et al. Inovação e Sustentabilidade: novos modelos e proposições. RAE -Revista de Administração de Empresas, v. 50, n. 2, p.146-154, abr./jun. 2010.

BARDIN, L. Análise de conteúdo. Lisboa: Edições 70, 2006.

BATISTA, A. V. et al. Plano Diretor Estratégico do Hospital Universitário Júlio

Bandeira - HUJB-UFCG. 2016. 141 f. Monografia (Especialização) - Curso de Gestão de Hospitais Universitários do Sus, Instituto Sírio Libanês de Ensino e Pesquisa, São Paulo, 2016.

BRASIL. Ministério da Saúde. Secretaria de Vigilância Sanitária. Portaria no 453, de 01 de junho de 1998. Aprova o Regulamento Técnico que estabelece as diretrizes básicas de proteção radiológica em radiodiagnóstico médico e odontológico, dispõe sobre o uso dos raios-X diagnósticos em todo território nacional e dá outras providências. Diário Oficial da União. Brasília, 02 jun. 1998.

BRASIL. Ministério do Meio Ambiente. Conselho Nacional do Meio Ambiente (CONAMA). Resolução $n^{\circ} 358$, de 29 de abril de 2005. Dispõe sobre o tratamento e a disposição final dos resíduos dos serviços de saúde e dá outras providências. Diário Oficial da União. Brasília, 04 maio 2005. n. 84, Seção 1, p. 63-65.

BUSS, P. M. et al. Governança em saúde e ambiente para o desenvolvimento sustentável. Ciência \& Saúde Coletiva, v. 17, n. 6, p. 1479-1491, 2012.

CAMPOS, F. J. B.; RAMOS, H. R. Aplicação do modelo TBL em um hospital público. Revista de Gestão Ambiental e Sustentabilidade, v. 3, n. 1. p. 124-138, jan./abr. 2014. 
CLARO, P. B. O.; CLARO, D. P.; AMÂNCIO, R. Understanding the sustainability concept in organizations. Revista de Administração, [S.1.], v. 43, n. 4, p. 289-300, dez. 2008.

DIAS, M. A. A. Resíduos dos serviços de saúde e a contribuição do hospital para a preservação do meio ambiente. Revista Academia de Enfermagem, v. 2, n. 2, p. 2129, jan. 2004.

EBSERH. Levantamento preliminar dos macroproblemas dos HUFs: Projeto Análise situacional de 12 HUFs. Brasília, 2016.

GIL, A. C. Métodos e Técnicas de Pesquisa Social. 4. ed. São Paulo: Atlas, 2008.

NASCIMENTO, E. P. Trajetória da sustentabilidade: do ambiental ao social, do social ao econômico. Estudos avançados, v. 26, n. 74, p. 51-64, 2012.

SACHS, I. Estratégias de transição para o século XXI: desenvolvimento e meio ambiente. São Paulo: Studio Nobel/Fundap, 1993.

SILVA, L. R. C. et al. Pesquisa documental: alternativa investigativa na formação docente. In: CONGRESSO NACIONAL DE EDUCAÇÃO - EDUCERE, 3., 2009, Curitiba. Anais... . Curitiba: [s.n], 2009. p. 4554-4566. Disponível em: www.pucpr.br/eventos/educere/educere2009/anais/pdf/3124_1712.pdf Acesso em: 15 maio 2017.

VILAÇA, W. P. T.; OLIVEIRA, M. M. Sustentabilidade e Comunicação no contexto hospitalar: estabelecendo a necessária conscientização. In: CONGRESO LATINOAMERICANO DE INVESTIGACIÓN DE LA COMUNICACIÓN, 9, 2008, Cidade do México. Anais. Cidade do México: Alaic, 2008. p. 1 - 15.

VILAS-BOAS, D. Sustentabilidade em estabelecimentos assistenciais de saúde soluções do projeto arquitetônico: soluções do projeto arquitetônico. Revista Interdisciplinar de Infraestrutura em Saúde, São Paulo, v. 8, n. 5, p.11-19, 2011.

WCED. World Comission on Enviromental And Development. Our common future. Oxford: Oxford University Press, 1987. 